\section{$\underset{\substack{\text { hommes } \\ \text { \& migrations }}}{ }$}

\section{Hommes \& migrations}

Revue française de référence sur les dynamiques

migratoires

$1309 \mid 2015$

Le $3 e$ âge des migrants

\title{
Awadi et la vieillesse en exil
}

\section{Bénédicte Maraval et Arnaud Veïsse}

\section{QpenEdition \\ Journals}

\section{Édition électronique}

URL : http://journals.openedition.org/hommesmigrations/3110

DOI : 10.4000/hommesmigrations.3110

ISSN : 2262-3353

\section{Éditeur}

Musée national de l'histoire de l'immigration

\section{Édition imprimée}

Date de publication : 1 janvier 2015

Pagination : 172-175

ISBN : 978-2-919040-30-8

ISSN : $1142-852 X$

\section{Référence électronique}

Bénédicte Maraval et Arnaud Veïsse, «Awadi et la vieillesse en exil », Hommes \& migrations [En ligne], 1309 | 2015, mis en ligne le 17 juillet 2015, consulté le 24 septembre 2020. URL : http:// journals.openedition.org/hommesmigrations/3110; DOI : https://doi.org/10.4000/ hommesmigrations.3110 


\section{AWADI ET LA VIEILLESSE EN EXIL}

BÉNÉDICTE MARAVALX, assistante sociale référente, et ARNAUD VEÏSSE, directeur général du Comité médical pour les exilés (Comede).

$E^{n}$ n février 2014, un monsieur peu sûr de lui se présente au centre de santé du Comede. Awadi est âgé de 74 ans. De nationalité comorienne, il réside en France depuis vingt-deux ans. II vit seul et n'a pas revu sa femme ni ses six enfants depuis son départ de Moroni. II nous explique avoir déjà consulté au Comede par le passé, ce qui est confirmé, en 2000, année de l'obtention de sa première carte de séjour pour raison médicale. Une fois régularisé, il a pu être affilié à l'assurance maladie, travailler comme ouvrier sur des chantiers, payer le loyer d'une chambre de foyer Sonacotra (Adoma, depuis 2007) et envoyer un peu d'argent au pays. II relevait donc logiquement du "droit commun". II s'est alors installé dans un quotidien en banlieue parisienne et petit à petit a trouvé des habitudes rassurantes dans un pays qui, maintenant, est le sien.

Pourtant, chaque année, à l'automne, commençait pour lui une période stressante : il devait redemander à son "praticien hospitalier" un "rapport médical sous pli confidentiel" pour pouvoir faire renouveler sa "carte de séjour temporaire". Il devait à nouveau faire la queue à la préfecture, fournir les mêmes documents que l'année pré- cédente, parfois un ou deux de plus, attendre la réponse et, dans son cas, être soulagé à la lecture du courrier l'autorisant à rester une année de plus en France. À l'automne 2013, il s'apprête à recommencer son parcours pour la treizième fois. Mais, cette fois, son médecin hospitalier l'a peut-être trouvé plus fatigué que les années précédentes, a pris plus de temps dans la consultation, ou il a rencontré une situation similaire peu de temps avant, quoi qu'il en soit, il lui conseille de retourner au Comede pour faire le point sur sa situation administrative. II suit ce conseil et trouve facilement le chemin.

\section{Un plan d'action pour l'accompagnement social}

Pour le service d'accueil contraint à "sélectionner" quotidiennement les personnes les plus "vulnérables" pour un suivi au centre de santé, Awadi n'est à première vue pas "prioritaire". II est suivi médicalement, n'a pas de rupture dans sa prise en charge, parle parfaitement français, a des revenus réguliers et, même s'il n'habite pas un 
trois pièces, il a un toit sur la tête. Mais, en poursuivant l'entretien avec le service social, il s'avère nécessaire de proposer à Awadi un rendez-vous pour affiner l'évaluation avant, éventuellement, de le ré-orienter vers un(e) assistant(e) social(e) de la mairie de son domicile ou du service hospitalier référent. Son âge avancé, son isolement, le fait qu'il réside dans un logement non adapté à sa situation, qu'il dépasse, de peu, les plafonds pour bénéficier de la complémentaire santé de la couverture maladie universelle (CMU), que son titre de séjour ne soit accordé que pour une durée de douze mois, qu'il n'ose pas se plaindre et qu'il ne connaisse pas ses droits le rendent vulnérable, invisible et précaire, comme tant d'autres. Awadi est ravi de la proposition et viendra au rendezvous, même si cela lui demande un gros effort puisqu'il lui faut plus d'une heure et demie de transport en commun pour cela.

Lors du premier rendez-vous, l'entretien se prolonge : Awadi semble avoir besoin d'expliquer sa situation, de s'excuser d'être là, de nous "prendre" autant de temps, tout en étant content de pouvoir "reprendre son histoire au Comede". Est-ce parce que c'est avec l'équipe du centre de santé qu'il a obtenu son premier titre de séjour ? Ou est-ce parce que enfin il peut parler, mettre des mots sur sa lassitude, parler de son sentiment d'être dans une impasse et de son angoisse pour son avenir ? Certainement un peu des deux et d'autres choses encore. Un plan d'action est décidé : d'abord, faire une demande de carte de résident de dix ans à la place de la carte de séjour temporaire d'un an, puis faire une demande d'aide à la complémentaire santé afin de pouvoir bénéficier d'une réduction sur les tarifs des mutuelles. Ensuite, faire une demande de logement social, insister sur la nécessité de déménager dans un appartement adapté rapidement. Enfin, Awadi tient à mettre sur cette liste la demande de nationalité française.

Il est prévenu que nous allons suivre ensemble les premières étapes de notre liste, mais que ce n'est pas forcément le Comede qui l'accompa- gnera dans les démarches suivantes. Les trajets sont longs et pénibles pour lui et nous pouvons l'orienter vers des professionnels de proximité. Nous sommes d'accord sur le principe. II repart de ce rendez-vous avec la liste des pièces à fournir pour une demande de carte de résident et un rendez-vous dix jours plus tard pour constituer son dossier de demande et prendre un courrier d'accompagnement. Nous convenons également qu'il ira à la préfecture pour obtenir un récépissé de renouvellement avant le prochain rendezvous, soit quelques jours avant la fin de l'expiration de son titre de séjour.

Au rendez-vous suivant, il s'est bien rendu à la préfecture, et il raconte avoir pleuré de stress devant l'agent du guichet. II a retrouvé la plupart des documents mais en a oublié certains chez lui, dans une enveloppe qu'il avait pourtant préparée. Avec l'accord d'Awadi, nous sollicitons de la directrice de son foyer une attestation de présence "dans le même bâtiment" depuis 2002 et l'informons de ses démarches. D'autres rendez-vous seront nécessaires pour compléter le dossier, au rythme de ses demandes de décaler les horaires pour des raisons de santé, parce qu'il devait se rendre au commissariat pour être entendu en tant que témoin d'une bagarre entre deux résidents, parce qu'il n'avait pas trouvé un document...

\section{De la carte de résident à la demande de naturalisation}

Au final, nous aurons tout de même réussi à réunir les 44 documents de preuve que nous avons joints au courrier. Entre-temps, la directrice du foyer nous alerte de l'interruption depuis plusieurs mois du versement de l'aide personnalisée au logement (APL) de la Caisse d'allocations familiales (CAF). Awadi avait oublié d'envoyer la copie de son récépissé de renouvellement. Nous le faisons aussitôt et la facture de loyer est mise 


\section{INITIATIVES}

en attente. Un jour de mai, Awadi arrive sans rendez-vous, très inquiet, une lettre de la préfecture à la main. Nous lisons le courrier ensemble : il lui est demandé de régler 241 euros +19 euros de timbres fiscaux afin de pouvoir retirer sa carte de résident. II repart rassuré et se rendra donc au rendez-vous à la préfecture (un peu) plus serein. Le mois suivant, il vient avec sa carte de résident et le formulaire de naturalisation qu'il a été chercher à la mairie. Cela semble primordial pour lui. Or il n'a pas réussi à trouver de l'aide dans sa ville. Nous nous reverrons plusieurs fois pour réunir l'ensemble des documents, en contactant les organismes concernés pour leur demander d'envoyer les attes-

Au-delà du cas d'Awadi,

la grande majorité de migrants âgés soignés et soutenus par le Comede cumulent les facteurs de vulnérabilité pour la santé. tations nécessaires. Dans l'intervalle, il faut à nouveau intervenir pour faire reprendre le versement des $A P L$, interrompu parce qu'il n'avait pas envoyé la copie de sa carte de résident. Lors des rendez-vous suivants, nous nous concentrerons sur la demande de logement et l'aide à la complémentaire santé. Pour limiter les trajets, il est convenu qu'il complétera lui-même les dossiers qu'on commence à constituer au centre de santé. Il se sent capable d'aller lui-même les déposer ou de les envoyer.

Six mois plus tard, nous nous revoyons pour faire le point sur sa situation. Il est sans nouvelles de la Sécurité sociale et de sa demande de logement. II n'a pas déposé sa demande de naturalisation car il n'a pas reçu l'attestation de la Caisse nationale d'assurance vieillesse (Cnav) demandée cinq mois plus tôt et censée arriver dans les dix jours suivant notre appel, et il ne voit pas comment il peut obtenir un certificat de décès de ses parents, morts depuis de très nombreuses années. De nouvelles interventions auprès des organismes concernés permettent de faire reprendre le cours des instructions.

Pour tous les exilés que nous accompagnons au Comede, l'administration est complexe et les démarches nombreuses pour accéder à un dispositif. Mais le "parcours du combattant" est encore plus difficile pour les personnes âgées, qui restent d'autant plus tributaires d'une situation de précarité administrative prolongée.

\section{Vulnérabilité multiple et épidémiologie médico-psycho-sociale}

Ainsi, pour Awadi, dont l'âge avance, la demande de nationalité devient pressante, alors que les démarches sont de plus en plus difficiles à accomplir. Dans un contexte d'isolement affectif et social et de perte progressive de son autonomie, il se sent débordé par toute démarche sortant du rythme quotidien. S'y ajoutent les problèmes de santé, de dépendance et de perte de mémoire, de logement inadapté, de faibles revenus, d'angoisse pour l'avenir, de distension des liens avec la famille restée au pays.

Au-delà du cas d'Awadi, la grande majorité de migrants âgés soignés et soutenus par le Comede cumulent les facteurs de vulnérabilité pour la santé ${ }^{2}$. Entre 2012 et 2014, plus de 700 personnes âgées de plus de 60 ans ont été accompagnées dans le cadre des permanences téléphoniques nationales (PT, 481) et au centre de santé à Bicêtre (CDS, 266). Elles vivent dans des conditions d'hébergement très précaires ( $98 \%$ des patients du CDS et $85 \%$ des bénéficiaires des PT n'ont pas de "chez soi") et disposent de ressources inférieures au plafond de la complémentaire CMU (98\% CDS, $91 \%$ PT).

Les patients du CDS sont en grande majorité 
hébergés chez un particulier (84\%, principalement un membre de famille), rarement en hébergement social (6\%), alors que $8 \%$ sont sans abri (3\% au Samu social, $1 \%$ dans un squat et $4 \%$ à la rue). La plupart d'entre eux n'ont pas (ou plus) de protection maladie ( $85 \%$ ), de même que la moitié des bénéficiaires des PT (49\%). Ils se trouvent en situation de séjour précaire ( $97 \%$ des patients et $84 \%$ des appelants), doublée d'un certain isolement social : $57 \%$ ne peuvent s'exprimer en français ou en anglais, et $9 \%$ n'ont personne pour les accompagner dans leurs démarches. Plus d'un patient sur dix n'a pas mangé à sa faim durant les derniers jours (12\%).

Parmi les autres facteurs de risque sur le plan de la santé mentale, on observe qu'une personne sur trois déclare avoir subi des violences par le passé, et près d'une sur dix des tortures. Sur le plan médical, outre les pathologies et handicaps associés au vieillissement précoce ${ }^{3}$, les maladies chroniques les plus fréquentes sont les maladies cardio-vasculaires, le diabète, l'infection chronique par le virus de l'hépatite $C$ et les cancers, ce qui nécessite la mise en place d'actions adaptées de dépistage, de bilans de santé et de prévention secondaire incluant des programmes d'éducation thérapeutique et le recours à l'interprétariat professionnel. Autant de prestations qui font souvent défaut dans les dispositifs de santé de droit commun.

Dans la diversité de leurs parcours migratoires et des motifs de leur exil (un tiers des migrants âgés suivis au centre de santé sont demandeurs d'asile), ces personnes ont un niveau d'instruction et de formation proche de celui des autochtones de la même génération (notamment sur le plan de la formation universitaire). Mais, comme de nombreux autres migrants âgés, leur situation de grande vulnérabilité sociale découle en premier lieu de la précarité administrative de leur statut. Dans de telles conditions, le "droit à bien vieillir" reste souvent hors d'atteinte, et les actions de soins et d'accompagnement sont essentielles pour corriger en partie les discriminations dont ils restent victimes.

\section{CONTACTER LE COMITÉ MÉDICAL POUR LES EXILÉS (COMEDE)}

Hôpital de Bicêtre

78, rue du Général-Leclerc BP31

94272 Le Kremlin-Bicêtre Cedex

\section{LES PERMANENCES NATIONALES DU COMEDE :}

- Permanence téléphonique droits, soutien, accès aux soins : 0145216312 (du lundi au jeudi 9 h 30-12 h 30)

- Permanence téléphonique médicale : 0145213893 (du lundi au vendredi de 14 h 30 à 17 h 30).

- Permanence téléphonique santé mentale : 0145213931 (les mardis et jeudis de 14 h 30 à 17 h 30). 